Article

\title{
A Novel Single-Axis MEMS Tilt Sensor with a High Sensitivity in the Measurement Range from $0^{\circ}$ to $360^{\circ}+$
}

Shudong Wang, Xueyong Wei * (1), Yinsheng Weng, Yulong Zhao and Zhuangde Jiang

State Key Laboratory for Manufacturing Systems Engineering, Xi'an Jiaotong University, Xi'an 710054, China; shudong_wang@outlook.com (S.W.); wengyinsheng@stu.xjtu.edu.cn (Y.W.); zhaoyulong@mail.xjtu.edu.cn (Y.Z.); zdjiang@mail.xjtu.edu.cn (Z.J.)

* Correspondence: seanwei@mail.xjtu.edu.cn; Tel.: +86-29-8339-5381

$+\quad$ This paper is an extended version of our paper published in Wang, S.; Ren, J.; Zhang, T.; Weng, Y.; Jiang, Z.; Wei, X. A MEMS Resonant Tilt Sensor with High Sensitivity Maintained in the Whole $360^{\circ}$ Measurement Range. In Proceedings of the 2016 IEEE SENSORS, Orlando, FL, USA, 30 October-3 November 2016; pp. 1159-1161.

Received: 16 November 2017; Accepted: 19 January 2018; Published: 25 January 2018

\begin{abstract}
In this paper, a novel single-axis MEMS tilt sensor is presented. It contains a hexagonal proof mass, six micro-lever force amplifiers and three double-ended-tuning fork (DETF) resonant strain gauges. The proof mass is placed in the center with the micro-levers and the DETFs radially arrayed around. The variation of gravity acceleration applied on the proof mass will result in frequency shifts of the DETFs. Angular tilt can be got by analyzing the frequency outputs. The structural design of the tilt sensor is optimized by finite element simulation and the device is microfabricated using a silicon-on-insulator process, followed by open-loop and closed-loop characterizations. Results show that the scale factor of such sensor is at least $11.53 \mathrm{~Hz} /$ degree. Minimum Allan deviation of the DETF oscillator is $220 \mathrm{ppb}$ (parts per billion) of the resonant frequency for an $5 \mathrm{~s}$ integration time. Resolution of the tilt sensor is $0.002^{\circ}$ in the whole measurement range from $0^{\circ}$ to $360^{\circ}$.
\end{abstract}

Keywords: double-ended tuning fork; MEMS; oscillator; resonator; tilt sensor

\section{Introduction}

Accurate tilt measurement is of great significance in many areas of civil, industrial and military uses. It has been utilized in somatic games, remote health monitoring for large structures, inertial navigations and etc. Therefore, many research groups are devoted to improve the performance of tilt sensors and related measuring systems [1-3]. Particularly, since the rapid development of MEMS technology, many kinds of sensors have been revolutionized and enabled a smaller dimension, a better performance and a lower price, leading to a rapid increase in the market share of sensors based on MEMS technology.

The main concept behind these MEMS tilt sensors is almost the same. First, there is a movable proof mass to sense the inertial force caused by gravity. This mass could be solid[4,5], liquid [6] or gas. Then there must be a physical response to the moving of the mass, which could be the change of capacitance [7], resistance [8] or resonance frequency [9-12]. An external circuit is needed to convert these responses to electrical signal, and angular tilt can be got through processing the electrical signal. Among those above, resonant MEMS tilt sensors are more attractive due to their quasi-digital output signal and high accuracy. Plenty of work have been devoted to improve the reliability and sensitivity of the resonant MEMS tilt sensors. Using differential structure is a common method to reduce the interference of temperature and residual stress [13]. One-stage or multi-stage micro-levers were 
employed and proved to be effective ways to amplify the inertial force as well as the sensitivity of tilt sensors [14,15]. In addition, mode localization effect in coupled micromechanical resonators was studied in MEMS and became a new sensing mechanism for tilt sensors [16]. Recently, a tilt sensor with a scale factor of $15.5 \mathrm{~Hz}$ /degree maintained in $\pm 20^{\circ}$ was reported, which is the highest among all [9]. Nevertheless, it is still hard to achieve a high sensitivity in a larger measurement range because of the sensor design and the related sensing mechanism. In this work, a novel MEMS tilt sensor is proposed to overcome the limited measurement range of high sensitivity. The sensing mechanism and structural design are first introduced, followed by open-loop and closed-loop characterizations. The experimental results indicate that the tilt sensor can maintain a high sensitivity in the whole measurement range from $0^{\circ}$ to $360^{\circ}$.

\section{Sensing Principle and Structure Design}

\subsection{Sensing Principle}

When a tilt angle is applied, sensing element of the tilt sensor will be affected by the gravity component, leading to a physical response, from which the tilt can be derived. In the case of MEMS resonance sensor, this physical response is frequency shift. Due to the direction of the gravity is always vertical to the ground, the physical response versus angle presents a sine function, causing a different sensitivity in different position.

Thus, tilt sensor made by a single element will have a limited measurement range of high sensitivity, generally $\pm 30^{\circ}$, in which the relation between tilt angle and output acceleration is approximately linear [17]. However, the range will be extended using a combination of three sensing elements, as shown in Figure 1 . When three sensing elements are radially arranged with $120^{\circ}$ apart from each other, there is always one sensing element more sensitive than the other two. Using data of the most sensitive element at any position, an accurate measurement of tilt in the whole range will be achieved accordingly. Łuczak gave the concept of tilt measurement systems utilizing multi-axial accelerometers [18], and disscussed application of arc tangent function, which can also ensure a constant sensitivity of tilt measurement [19]. Enfu Li et al. achieved a tilt sensor with a high resolution maintained in the full measurement range based on one dual-axis micro-accelerometer [20]. In our previous work, we built a tilt measurement system based on three accelerometers, whose resolution is $0.02^{\circ}$ [3]. In this work, we will integrate three sensing elements in MEMS process, and propose a MEMS tilt sensor with a smaller size, and a better performance.

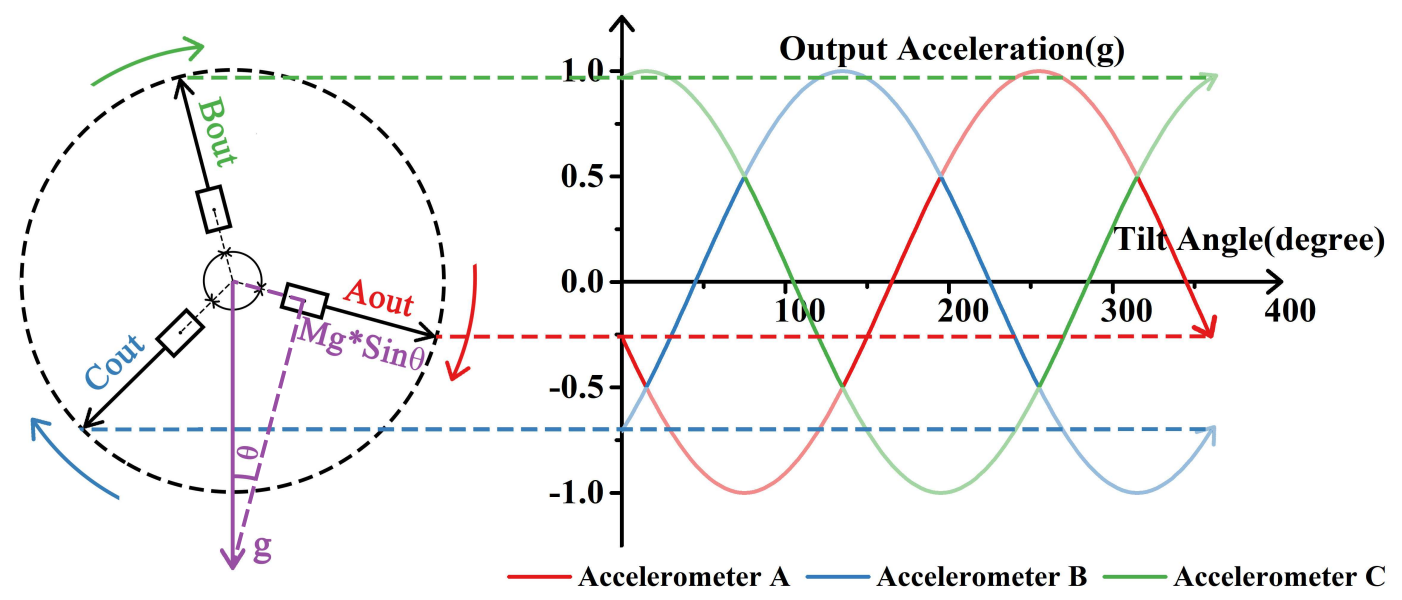

Figure 1. Schematic drawing of tilt measurement based on three accelerometers and simulated output of each accelerometer as rotating. 


\subsection{Sensor Design}

Based on aforementioned sensing principle, a novel single-axis MEMS tilt sensor is thus proposed as shown in Figure 2. It contains a hexagon proof mass, six micro-lever force amplifiers and three DETFs. The proof mass is placed in the middle with the micro-levers and the DETFs radially arrayed around, with a $120^{\circ}$ apart from each other, as discussed above. All of the DETFs are connected to the proof mass through the micro-levers. Under working conditions, the tilt sensor is placed in the vertical plane. When the proof mass displaces due to the gravity, different components of gravity will apply on each micro-lever, which amplifies the force exerted on the DETFs. As a result, the resonant frequencies of DETF resonators will be tuned [12] and can be expressed as follow:

$$
f=f_{n} \sqrt{1+\frac{0.293 P l^{2}}{E t w^{3}}}
$$

where $f_{n}$ is the characteristic frequency of the DETF, $l, w$ and $t$ indicate the length, width and thickness of each resonant beam respectively. $P$ is the axial force applied on the resonant beam, which is a negative number when compressive stresses applied and it is a positive number when tensile stresses applied, E represents the Young's modulus of silicon.

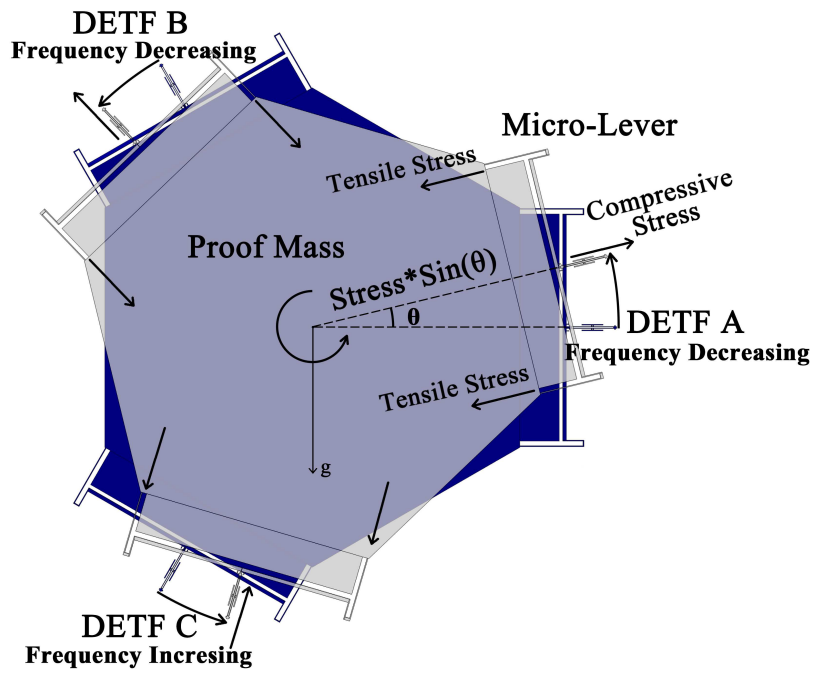

Figure 2. Structure of the tilt sensor.

Method of finite element analysis was used to aid the design of the structure and find the optimum dimensions. Relationship between frequency shift and tilt angle can be easily botained as well in the simulation. Accordingly, the scale factor of tilt sensor is calculated by:

$$
S=\frac{\Delta f}{\Delta \theta}
$$

where $\Delta f$ is the variation of DETF's resonant frequency and $\Delta \theta$ is the inclination of the whole device. Note that scale factor is a variate according to the angular position, and it remains high-level in $\pm 30^{\circ}$, which is also called "sensitive region".

In order to maximize $\Delta f$, six micro-lever force amplifiers were used to magnify the force applied on each DETF. The micro-lever conprises an input beam, a lever beam, a pivot beam and an output beam [21]. The input beam was designed to be long and in folded form, thus minimize the effect of the component gravitational acceleration perpendicular to the axis of the DETFs, also known as "crosstalk". The optimized geometry dimensions of the tilt sensor are given in Table 1. Figure 3 
shows the vibration mode of DETF B (a), and the simulation results of its resonant frequency versus inclination (b). The approximate linear interval and sensitive region is marked in the figure.

(a)

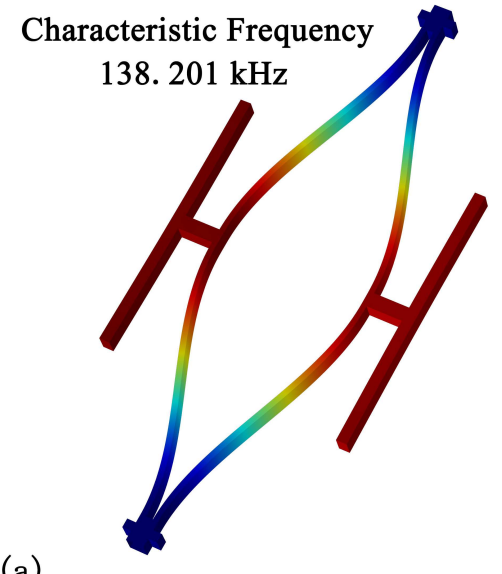

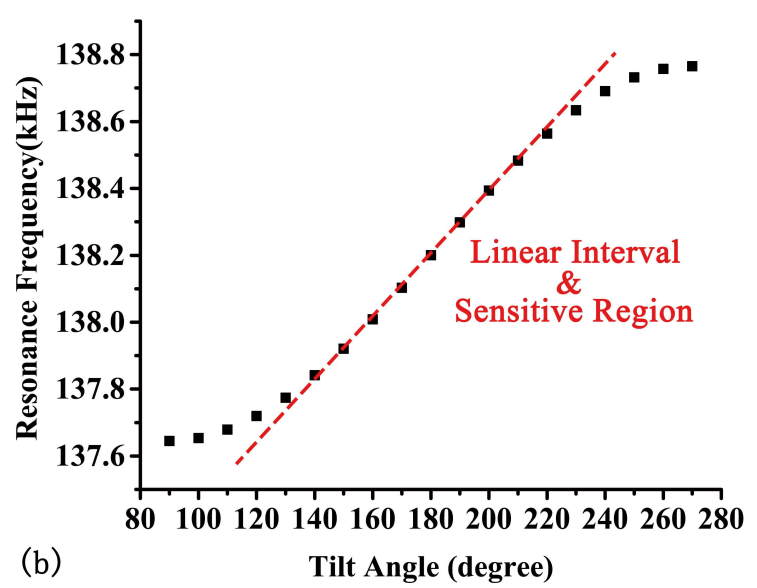

(b)

Tilt Angle (degree)

Figure 3. Vibration mode of DETF B (a), and its resonant frequency versus inclination (b).

Table 1. The geometry dimensions of DETF resonators.

\begin{tabular}{cc}
\hline Resonant beam length & $300 \mu \mathrm{m}$ \\
Resonant beam width & $3 \mu \mathrm{m}$ \\
Device thickness & $10 \mu \mathrm{m}$ \\
Die area & $5.5 \mathrm{~mm} \times 5.5 \mathrm{~mm}$ \\
DETF A frequency & $151.815 \mathrm{kHz}$ \\
DETF B frequency & $138.201 \mathrm{kHz}$ \\
DETF C frequency & $138.211 \mathrm{kHz}$ \\
\hline
\end{tabular}

Due to the anisotropy properties of single-crystal-silicon being used, two of the DETFs are of the same resonant frequency due to their similar crystal orientation on the silicon wafer, while the other one is far different. Thus a normalization of their frequencies is required to identify the DETF that is in the most sensitive region:

$$
f_{\text {norm }}=\frac{f_{M}-f_{\text {avg }}}{\frac{1}{2} f_{p p}}
$$

where, $f_{M}$ is the measured frequency of each DETF, $f_{\text {avg }}$ is the average of the frequencies in the whole measurement range, $f_{p p}$ is the peak-to-peak value of frequencies.

After normalization, frequencies of the three DETFs will transform into three standard sine waves, as shown in Figure 1, and the most sensitive DETF at each given position can be clearly found out. Thus, the angle $\theta$ can be got and the formula is:

$$
\theta=\sin ^{-1}\left(f_{\text {norm }}\right)
$$

where $f_{\text {norm }}$ is the normalized output frequency of the most sensitive one of the three DETFs at the given position.

Detailed information was given in our previous work [21]. As a result, the simulated sensitivities of DETF A, B and C are $10.38 \mathrm{~Hz} /$ degree, $9.34 \mathrm{~Hz} /$ degree and $9.33 \mathrm{~Hz}$ /degree respectively. Therefore, the scale factor of the sensor in the measurement range from $0^{\circ}$ to $360^{\circ}$ is theoretically no less than $9.33 \mathrm{~Hz} /$ degree. 


\subsection{Temperature Drift}

Single-crystal silicon is an anisotropic material whose elasticity matrix and Young's modulus change as temperature varies. In this way, effect of external temperature should be considered and compensated. In order to quantify this effect, finite element analysis method was used and the results are shown in Figure 4. Thermal expansion was assumed to be negligible in this simulation model.
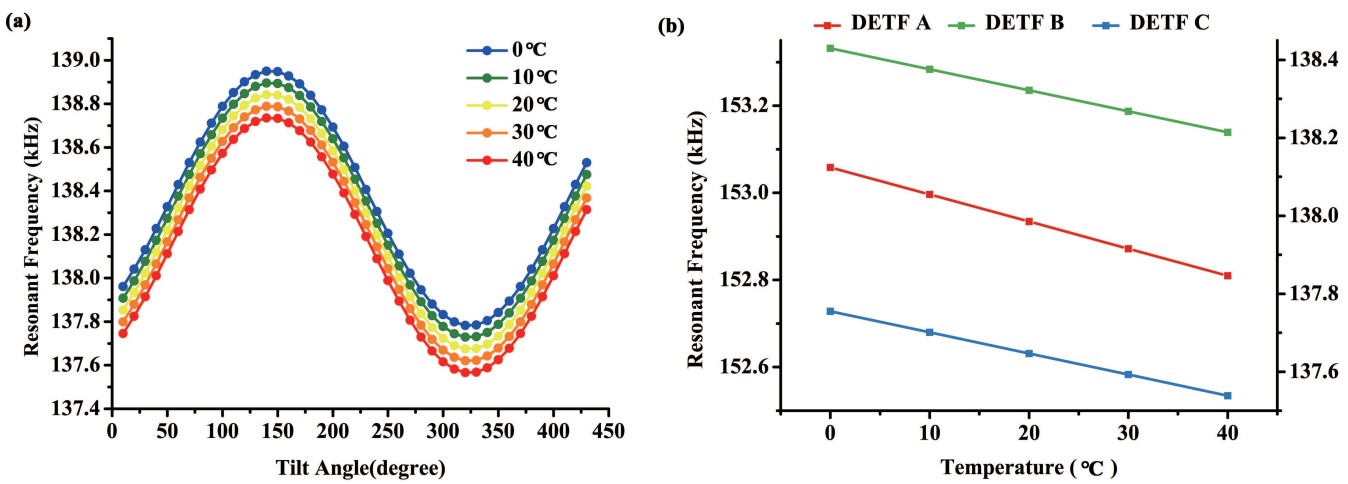

Figure 4. Resonant frequency of DETF B in different temperature environments vs. tilt angle (a) and resonant frequency of the three DETFs at a given position vs. temperature (b).

Figure 4a demonstrates resonant frequency of DETF B in different temperature environments versus tilt angle. As the temperature rises, characteristic frequency of the DETF reduces, while the scale factor remains the same. Figure $4 \mathrm{~b}$ shows the linear relation between resonant frequency and ambient temperature. The fitted slopes of the three lines are $6.21 \mathrm{~Hz} /{ }^{\circ} \mathrm{C}, 5.4 \mathrm{~Hz} /{ }^{\circ} \mathrm{C}$, and $5.4 \mathrm{~Hz} /{ }^{\circ} \mathrm{C}$ respectively. The differences among them may caused by different crystal orientations.

Temperature drift will not affect scale factor or resolution of the tilt sensor, however, it will reduce stability of the frequency data and effect the result of angle calculation. Therefore, in practical use, influence of external temperature should be compensated [9,22].

\section{Experimental Results of Open-Loop Test}

The described tilt sensor was microfabricated using a standard SOI-MEMS technology and the mechanical layer thickness of the SOI is $10 \mu \mathrm{m}$. A micrograph of the tilt sensor and its package is shown in Figure 5. Three DETF resonators are separated $120^{\circ}$ apart and connect to a common proof mass in the center through three pairs of lever amplifiers.

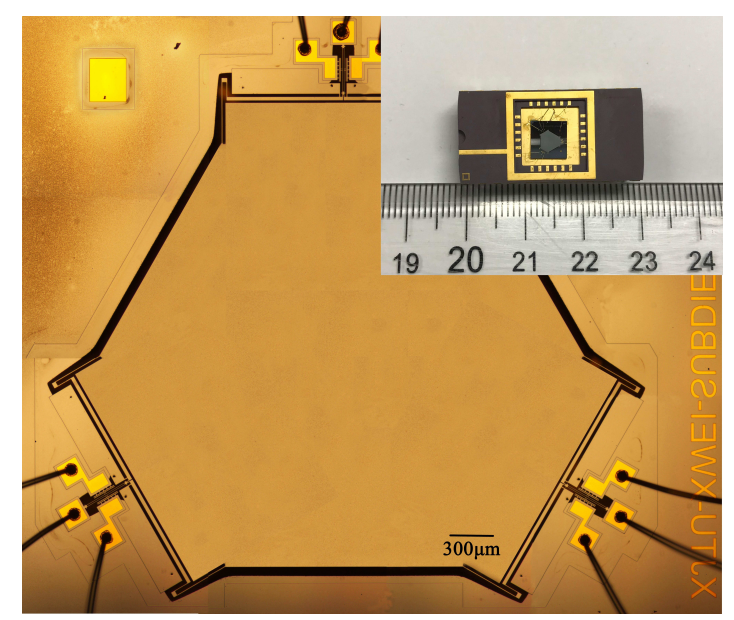

Figure 5. Micrograph of the tilt sensor and package of the device. 
Figure 6 shows the schematic diagram of the open-loop test circuit (a) and the testing setup (b). The tilt sensor was mounted in the vertical plane on a rotary table, which is placed in a vacuum chamber. The rotary table was operated by an external controller, and its positionaI accuracy was $0.01^{\circ}$. The vacuum level was settled to $10 \mathrm{~Pa}$ thus the effect of air damping was reduced and a high Q-factor of the DETF was achieved. When the rotary table rotates, the three DETFs are subject to different component gravitational acceleration as illustrated in Figure 2.
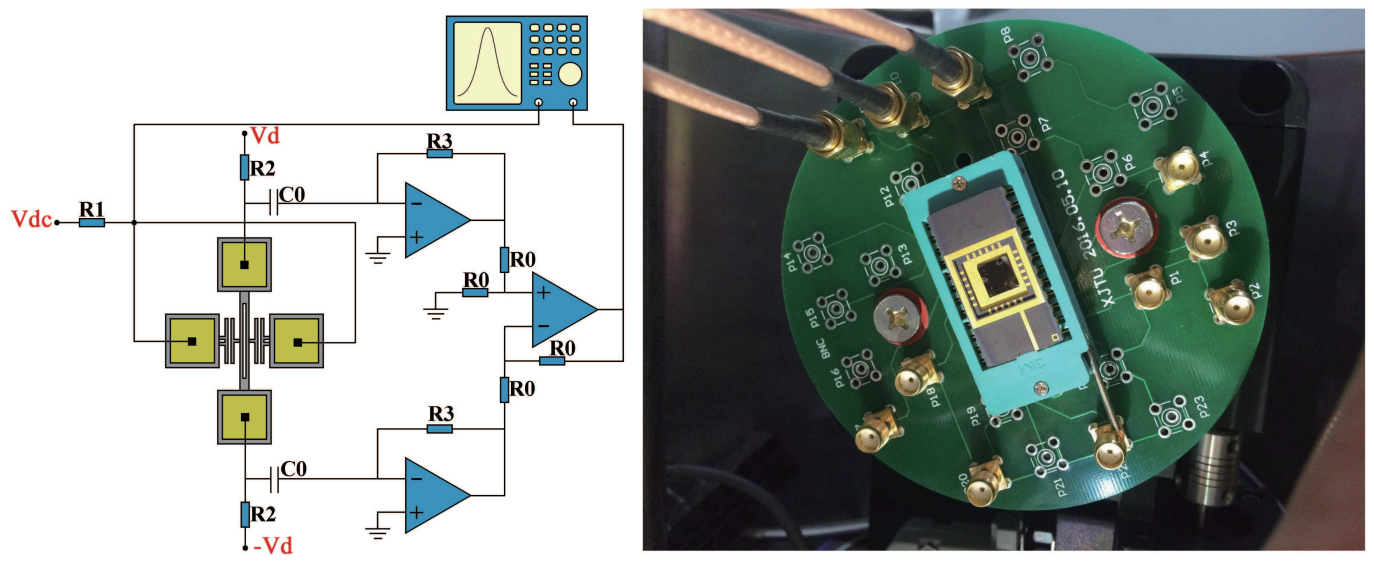

Figure 6. Schematic diagram of the open-loop test circuit (a) and installation (b).

In this experiment, the DETF resonator is electrically driven and sensed through parallel electrodes. A DC source (KEYSIGHT E3649A) was used to provide DC-bias on the electrodes. A network analyzer (KEYSIGHT E5061B) was also applied to characterize the frequency responses of the three DETFs. A differential piezoresistive sensing scheme was used to achieve a better feedthrough cancellation and a higher signal-noise ratio [23]. Figure 7 shows the vibration amplitude of DETF B obtained from the network analyzer when the device is tilted from $-70^{\circ}$ to $70^{\circ}$ at a step of $10^{\circ}$. The resonant frequency of DETF $B$ is tuned as the rotary table rotates, and the frequency shift is larger within the range from $-30^{\circ}$ to $30^{\circ}$, which is the sensitive region of DETF B as indicated by Figure 1.

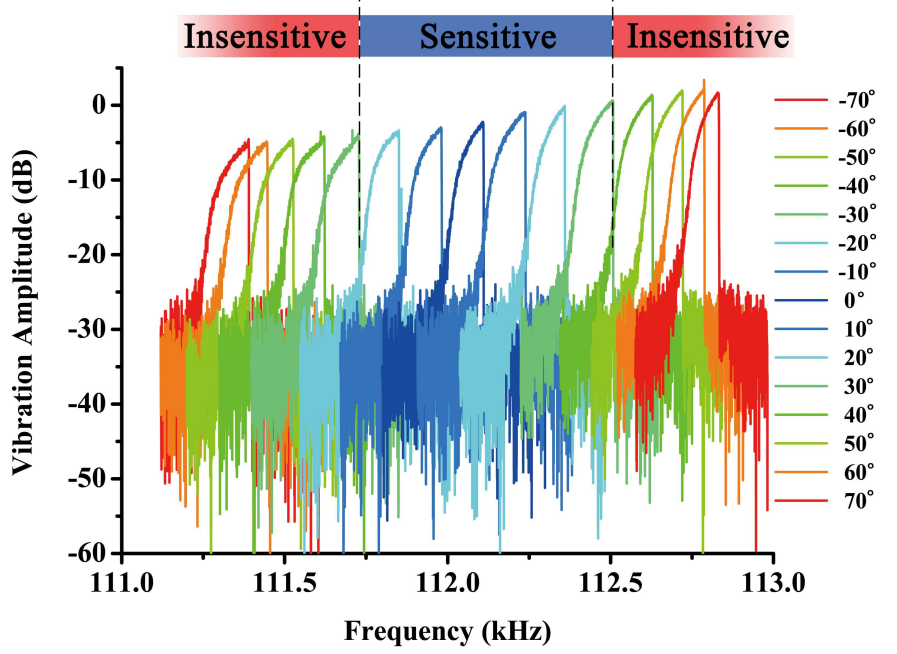

Figure 7. Open-loop test result of DETF B from $-50^{\circ}$ to $50^{\circ}$.

The frequency response curves in Figure 7 indicate that the DETF is oscillating in its nonlinear vibration state. As the driving frequency sweeps upward and downward, a hysteresis region will be observed with two jumping points in the frequency response curves [24]. Both frequencies at 
the jumping points are subject to axial stress tuning of DETF. In the experiment, we found that the frequency at the lower jumping point is more stable than the peak frequency at the upper jumping point as the sensor tilts. Therefore, the frequency data was recorded while the rotary table rotates from $0^{\circ}$ to $360^{\circ}$ with a step of $10^{\circ}$ and the experiment was done in a rather stable vacuum pressure and ambient temperature.

Figure 8a shows the resonant frequencies of three DETFs versus tilt angle and accordingly there are three sine curves of frequencies with phase differences of $120^{\circ}$ as predicted in Figure 1 . Due to the anisotropic properties of single crystal silicon and the unavoidable processing error, the characteristic frequencies of the micro-fabricated DETFs slightly differ from the FEA simulation results and vary from each other. The frequency response of each DETF in the sensitive region is extracted and shown in Figure 8b, $\mathrm{c}$ and $\mathrm{d}$ respectively. After a linear fitting of the data, the scale factors of three DETFs are $12.73 \mathrm{~Hz} /$ degree, $11.84 \mathrm{~Hz} /$ degree and $11.53 \mathrm{~Hz}$ /degree respectively. Therefore, the scale factor of the tilt sensor is no less than $11.53 \mathrm{~Hz} /$ degree.
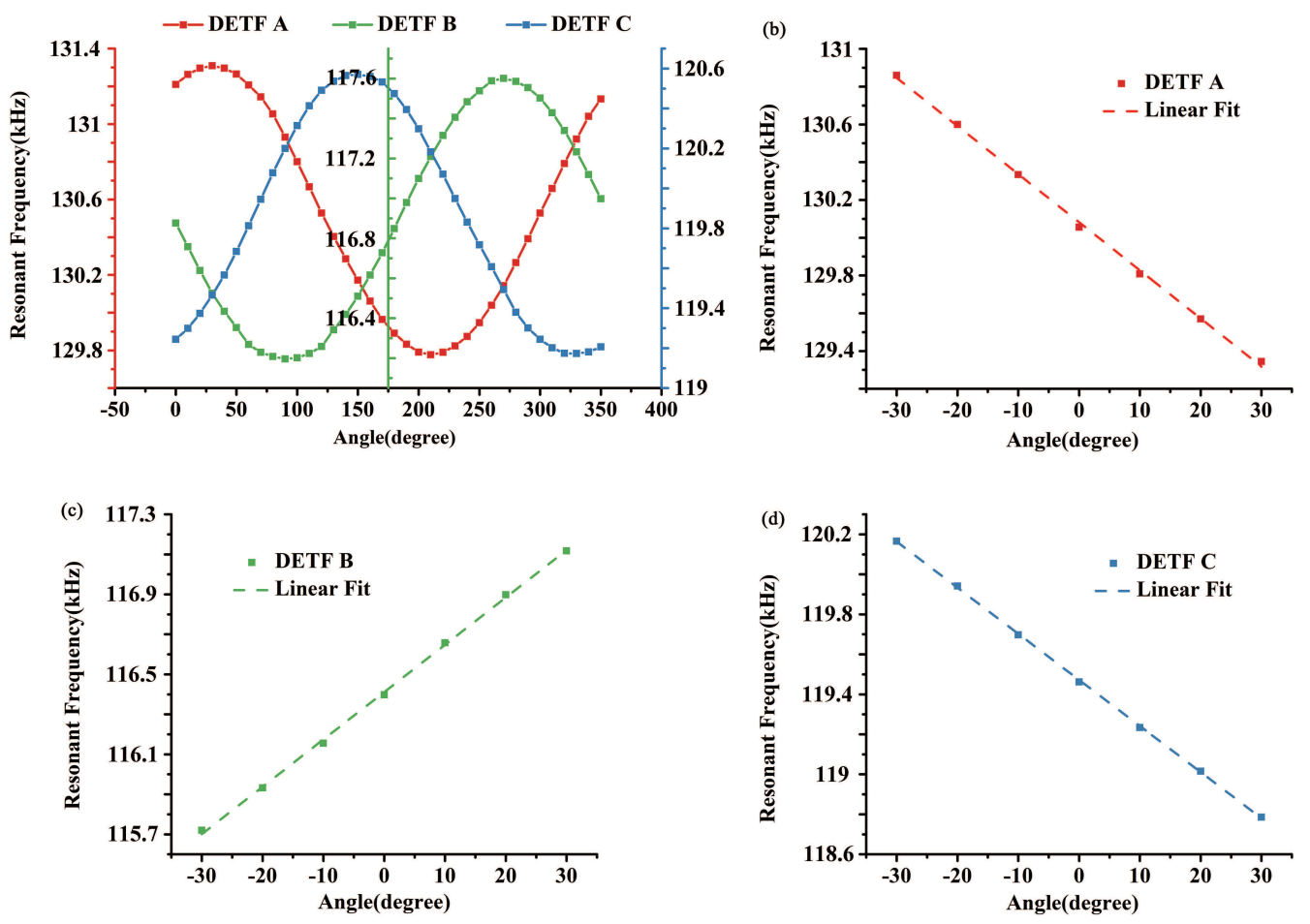

Figure 8. (a) Resonant frequency of the three DETFs got by downward sweeps; (b) Frequency of DETF A in sensitive region; (c) Frequency of DETF B in sensitive region; (d) Frequency of DETF C in sensitive region.

Three DETFs were designed to be $120^{\circ}$ apart from each other, however, the angles between each DETF are slightly different from $120^{\circ}$ due to manufacturing process error. These angular misalignments can be obtained by analyzing the normalized frequency of the three DETFs derived from (5). As Figure 9 shows, after measuring and fitting the data, it was found that $\theta a b$ is $119.8^{\circ}, \theta b c$ is $119.54^{\circ}$, and $\theta a c$ is $120.66^{\circ}$. These errors will be considerded in the future analysis. 


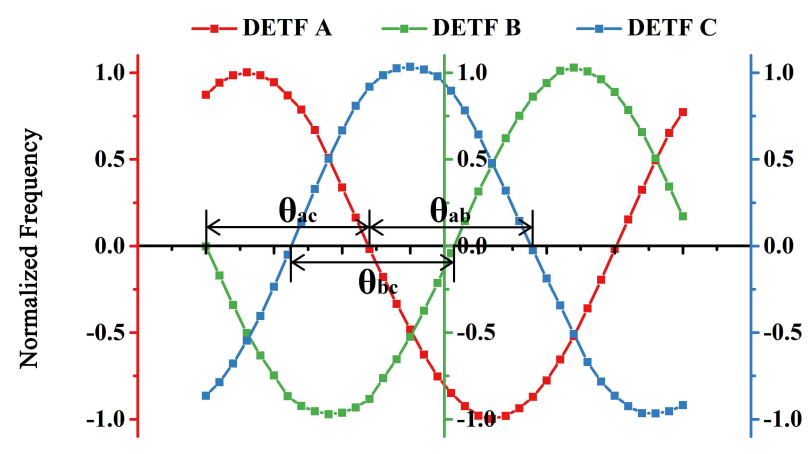

Figure 9. Normalized Frequency of the three DETFs.

\section{Experimental Results of Closed-Loop Test}

The closed-loop tests are suitable for practical application field, and it is the first step of industrialization of MEMS resonant sensors. In the closed-loop tests, micromechanical oscillators based on the DETF resonators were built up as shown in Figure 10. The oscillation circuit contains a differential piezoresistive sensing circuit, a band pass filter, a phase shifter and a comparator. The DETF is electrostatically actuated in its anti-phase vibration mode and its transverse displacement is detected through the differential piezoresistive sensing method. The resulting signal is filtered and phase shifted before entering into comparator. The output signal of comparator together with a DC bias is applied on the driving electrodes to excite the DETF resonator. The time-varying feedback force compensates the energy dissipation and this closed loop feedback sustains the oscillation of DETF. The oscillation frequency changes as the sensor tilts, which ensures a practical measurement of tilt angle.

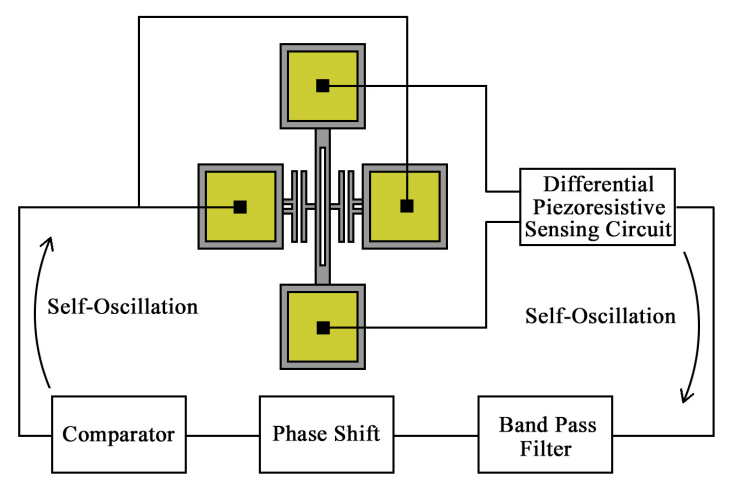

Figure 10. Micromechanical oscillator based on the DETF resonator.

In the static test, the tilt sensor was mounted still on the vertical plane. The frequency output was recorded by a frequency counter for $20 \mathrm{~min}$ with a $10 \mathrm{~ms}$ gate time. A short gate time ensures frequency data being rapidly read-out, but also leads to a relatively high systematic noise caused by the frequency counter. Therefore, trade off between quick response and high stability should be considered in practical use.

The measured sensor signal is usually affected by different factors such as quantization noise, velocity random walk, bias instability, etc. [25]. As shown in Figure 11a, the output frequency of DETF $\mathrm{B}$ oscillator is very noisy and the frequency fluctuation is up to $3 \mathrm{~Hz}$, which dramatically degrades the performance of tilt sensor. Kalman Filter (KF) was found to be an adequate way to reduce noise and improve resolution of the system. Comparing to moving-average filters, Kalman Filter enables the tilt sensor a better dynamic response and adaptability of complex testing environments [26]. In order 
to improve the filtering effect while reducing the delay time caused by KF, we have found the most appropriate model parameters in our previous work [2].

Allan deviation method was also used to study the frequency stability of oscillator circuit. As indicated in Figure 11b, KF can reduce the short-time noise remarkably as R increases. However, it is incapable of canceling long-term drift and accordingly Allan deviation of the processed data are the same as the integration time gets longer. Minimum Allan deviation of the origin data is found to be $220 \mathrm{ppb}$ at $5 \mathrm{~s}$, and the resolution of our tilt sensor can be got by:

$$
R=\frac{A \times f_{0}}{S}
$$

where $A$ is the minimum Allan deviation of the data, $f_{0}$ is the characteristic frequency of the DETF, $S$ is the scale factor of the tilt sensor. In this way, resolution of the tilt sensor is $0.002^{\circ}$.
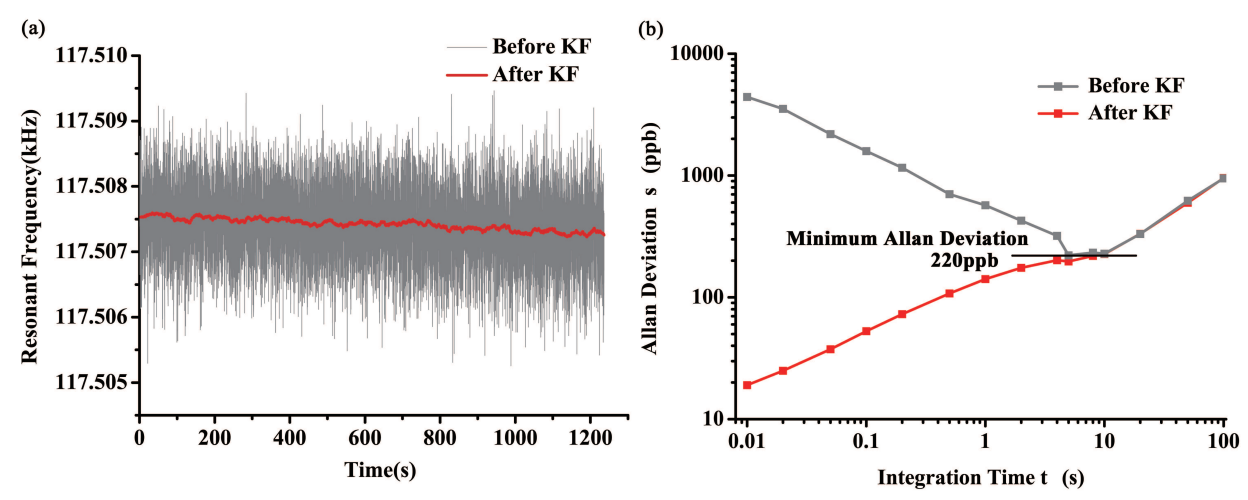

Figure 11. (a) Static frequency data of DETF B before and after Kalman Filter; (b) Allan deviation of the static frequency data with different parameter $\mathrm{R}$.

Resolution of the sensor was also experimentally measured. Installation of the laboratory apparatus and the tilt sensor was the same as the open-loop test. DETF B was embedded in the self-oscillation circuit to read out its resonant frequency in real time while the rotary table was working. The sampling interval of the frequency data was $100 \mathrm{~ms}$. Positional accuracy of the rotary table is $0.01^{\circ}$, so the minimum angle variation was set to be $0.01^{\circ}$. Figure 12 shows the performance of the tilt sensor in the resolution test. Frequency shift between each step was about $0.13 \mathrm{~Hz}$, which coincide with the scale factor measured in the open-loop test. Response time of the sensor is measured to be about $2 \mathrm{~s}$. As a result, it can be found that resolution of the tilt sensor is better than $0.01^{\circ}$.

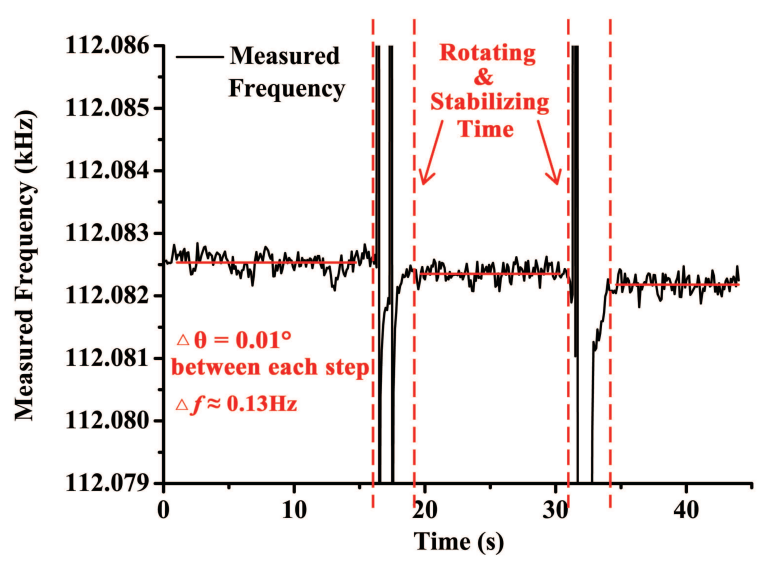

Figure 12. Resolution test of the tilt sensor. 
Accuracy is another feature to evaluate the performance of the tilt sensor, which is measured as a highest deviation of a value represented by the sensor from the ideal or true value. It can be estimated by the following formula:

$$
A=\sqrt{N^{2}+H^{2}+R^{2}}
$$

where $N$ is the nonlinearity of the output, $H$ is its hysteresis, and $R$ is its repeatability. To calibrate these characteristics, DETF B was tested repeatedly in the range from $-30^{\circ}$ to $30^{\circ}$ with a step of $10^{\circ}$. During each step, the rotary table was still for $5 \mathrm{~s}$ and average of the frequency datum were recorded. Output of DETF B at each position is given in Table 2.

Table 2. Output of DETF B at each position.

\begin{tabular}{cccccccc}
\hline \multirow{2}{*}{ Tilt Angle (degree) } & Acceleration (g) & \multicolumn{5}{c}{ Frequency Data (Hz) } \\
\cline { 3 - 7 } & & 1st & 2nd & 3rd & 4th & Average & Standard Deviation \\
\hline-30 & -0.5 & $112,592.6$ & 112,592 & $112,590.3$ & $112,589.6$ & $112,591.1$ \\
-20 & -0.342 & $112,470.7$ & $112,469.4$ & $112,473.1$ & $112,468.7$ & $112,470.5$ & 1.2 \\
-10 & -0.174 & $112,337.9$ & $112,337.6$ & $112,340.3$ & 112,337 & $112,338.2$ & 1.6 \\
0 & 0 & $112,198.4$ & $112,199.9$ & 112,202 & $112,198.9$ & $112,199.8$ & 1.2 \\
10 & 0.174 & $112,059.4$ & 112.061 .1 & $112,061.6$ & $112,060.5$ & $112,060.7$ & 0.8 \\
20 & 0.342 & $111,921.7$ & $111,923.4$ & $111,924.3$ & $111,923.8$ & $111,923.3$ \\
30 & 0.5 & $111,791.2$ & $111,793.2$ & $111,793.8$ & $111,793.3$ & $111,792.9$ \\
\hline
\end{tabular}

After a linear fitting of the data as shown in Figure 13, transfer function of DETF B can be described as:

$$
y=-798.67 x+112196.6
$$

where $y$ is the frequency data, and $x$ is the applied acceleration. Maximum regular residual was found to be $4.83 \mathrm{~Hz}$ at the position of $-30^{\circ}$, which corresponds to $-0.5 \mathrm{~g}$. Thus the nonlinearity can be derived by:

$$
N=\frac{Y_{\text {Nmax }}}{Y_{F S}} \times 100 \% \text { FS }=\frac{4.83 \mathrm{~Hz}}{798.7 \mathrm{~Hz}} \times 100 \% F S=0.61 \% \text { FS }
$$

where $Y_{\text {Nmax }}$ is the maximum regular residual, $Y_{F S}$ is the full scale frequency shift, which in this experiment $\left( \pm 30^{\circ}\right.$, which corresponds to $\left.\pm 0.5 \mathrm{~g}\right)$ is $798.7 \mathrm{~Hz}, F S$ represents full scale of the measurement range.

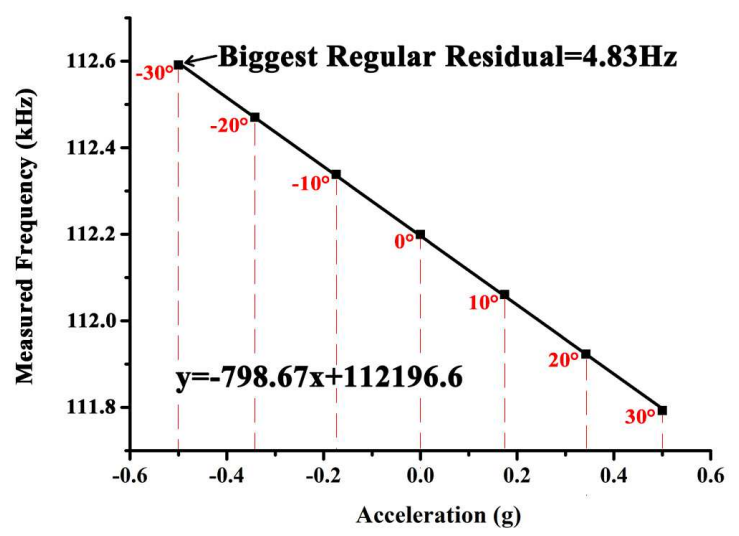

Figure 13. Linear fit of the frequency data. 
Hysteresis of the sensor represents the inconsistency of its output during loading and unloading. It can be seen from the experimental data that the maximum deviation is $3.5 \mathrm{~Hz}$, at the position of -20 degree. Thus the hysteresis can be got by:

$$
H=\frac{Y_{H \max }}{2 \times Y_{F S}} \times 100 \% \text { FS }=\frac{3.5 \mathrm{~Hz}}{2 \times 798.7 \mathrm{~Hz}} \times 100 \% \text { FS }=0.22 \% \text { FS }
$$

where $Y_{\text {Hmax }}$ is the maximum difference of the output.

The repeatability represents inconsistency of the measured results in multiple experiments. It can be derived by:

$$
R=\frac{c \times S_{a v}}{Y_{F S}} \times 100 \% F S=0.46 \% \text { FS }
$$

where $c$ is the confidence factor, and in this four-back-and-forth experiment $c=3.182, S_{a v}$ is the standard deviation of the measured data.

To sum up, the nonlinearity of the tilt sensor is $0.61 \% \mathrm{FS}$, the hysteresis is $0.22 \% \mathrm{FS}$, and the repeatability is $0.46 \%$ FS. According to the fuluma above, accuracy of the tilt sensor is calculated to be $0.79 \%$ FS.

\section{Conclusions}

This paper reports a novel resonant MEMS tilt sensor of high sensitivity maintained in an expanded measurement range of $0^{\circ} \sim 360^{\circ}$. The tilt sensor is microfabricated using a standard SOI technology. Open loop testing results show that the presented sensor can achieve a sensitivity of $11.53 \mathrm{~Hz} /$ degree in the full scale range. Closed-loop oscillator circuit has been built up and characterized by using Allan deviation method. The testing results show that the resolution of the tilt sensor is $0.002^{\circ}$, and the accuracy is $0.79 \%$ FS. The proposed accelerometer was compared with others from publications in the measurement range, die area, scale factor, resolution and accuracy together. The comparison results were shown in Table 3. These values compare well with other reported MEMS tilt sensors.

Table 3. Comparison between this work and other reported.

\begin{tabular}{cccccc}
\hline & Range (degree) & Die Area $(\mathbf{m m} \times \mathbf{m m})$ & Scale Factor & Resolution & Accuracy \\
\hline Our work & \pm 180 & $5.5 \times 5.5$ & $11.53 \mathrm{~Hz} /$ degree & $0.002^{\circ}$ & $0.79 \% \mathrm{FS}$ \\
{$[4]$} & \pm 20 & $4.5 \times 4$ & $24.5 \mathrm{~Hz} /$ degree & $0.00003^{\circ}$ & $/$ \\
{$[13]$} & \pm 180 & $13 \times 13$ & $6.317 \mathrm{~Hz} / \mathrm{g}$ & $0.76^{\circ}$ & $0.67 \% \mathrm{FS}$ \\
{$[27]$} & \pm 90 & $7 \times 7$ & $45 \mathrm{ppm} /$ degree & $/$ & $/$ \\
\hline
\end{tabular}

Future work will focus on further increasing scale factor and resolution of the tilt sensor. Integrated packaging of the whole system will be the main content of the research. Also, compensation of temperature drift and other environmental factors will be studied.

Acknowledgments: This work is supported by the National Natural Science Foundation of China (51575439 and 51421004), Natural Science Foundation of Shaanxi Province (2014jm2-5054) and 111 project (B2016). The authors also appreciate the support from the Collaborative Innovation Center of High-End Manufacturing Equipment and the International Joint Laboratory for Micro/Nano Manufacturing and Measurement Technologies.

Author Contributions: Xueyong Wei and Shudong Wang conceived the research and designed the sensor. Shudong Wang and Yinsheng Weng performed the experiments and analyzed the data. Shudong Wang, Yinsheng Weng and Xueyong Wei wrote the paper. Yulong Zhao, Zhuangde Jiang and Xueyong Wei supervised the work.

Conflicts of Interest: The authors declare no conflict of interest. 


\section{References}

1. Bajic, J.; Stupar, D.; Manojlovic, L.; Slankamenac, M.; Zivanov, M. A simple, low-cost, high-sensitivity fiber-optic tilt sensor. Sens. Actuators A 2012, 185, 33-38.

2. Weng, Y.; Zhang, H.; Ren, J.; Wang, S.; Wei, X. Dynamic Performance of A Novel Tilting Angle Measurement System Using Three Accelerometers. In Proceedings of the 2016 IEEE Sensors, Orlando, FL, USA, 30 October-2 November 2016; pp. 940-942.

3. Weng, Y.; Wang, S.; Zhang, H.; Gu, H.; Wei, X. A High Resolution Tilt Measurement System Based on Multi-accelerometers Measurement 2017, 109, 215-222.

4. Chang, C.-T.; Tsai, M.-H.; Liu, Y.-C.; Sun, C.-M.; Fang, W. Design and implementation of an extremely large proof-mass COMS-MEMS capacitive tilt sensor for sensitivity and resolution improvement. In Proceedings of the 2011 16th International Solid-State Sensors, Actuators and Microsystems Conference (TRANSDUCERS), Beijing, China , 5-9 June 2011; pp. 1104-1107.

5. Liu, H.; Pike, W.T. A micromachined angular-acceleration sensor for geophysical applications. Appl. Phys. Lett. 2016, 109, 173506 .

6. Liu, S; Zhu, R. Micromachined Fluid Inertial Sensors. Sensors 2017, 17, 367.

7. Hu, P.; Guo, J.; Tan, J. An Annular Planar-capacitive Tilt Sensor with a $360^{\circ}$ Measurement Range. IEEE Trans. Ind. Electron. 2016, 63, 2469-2476.

8. Zhang, W.; Zhu, H.; Lee, J.E.Y. Piezoresistive transduction in a double-ended tuning fork SOI MEMS resonator for enhanced linear electrical performance. IEEE Trans. Electron Devices 2015, 62, 1596-1602.

9. Zou, X.; Thiruvenkatanathan, P.; Seshia, A.A. A high-resolution micro-electro-mechanical resonant tilt sensor. Sens. Actuators A Phys. 2014, 220, 168-177.

10. Alves, F.S.; Dias, R.A.; Cabral, J.M.; Gaspar, J.; Rocha, L.A. High-Resolution MEMS Inclinometer Based on Pull-In Voltage. J. Microelectromech. Syst. 2012, 24, 931-939.

11. Sato, K.; Ono, A.; Tomikawa, Y. Experimental study of gyro sensor using double-ended tuning fork quartz resonator. In Proceedings of the 2004 IEEE International on Frequency Control Symposium and Exposition, Montreal, QC, Canada, 23-27 August 2004; pp. 575-578.

12. Abdolvand, R.; Bahreyni, B.; Lee, J.E.Y.; Nabki, F. Micromachined resonators: A review. Micromachines 2016, $7,160$.

13. Li, C.; Zhao, Y.; Li, B.; Cheng, R.; Sun, D.; Han, C.; Zhao, Y. A micro-machined differential resonance accelerometer based on silicon on quartz method. Sens. Actuators A Phys. 2014, 253, 1-9.

14. Ding, H.; Zhao, J.; Ju, B.; Xie, J. A highly-sensitivity biaxial resonant accelerometer with two-stage microleverage mechanisms. J. Micromech. Microeng. 2016, 26, 015011.

15. Cheng, L.; Yue, W.; Shangchun, F.; Baoxi, K.; Chao, W. Design of a new differential silicon resonant accelerometer with dual proofmasses using two-stage microlever. In Proceedings of the 2015 IEEE on SENSORS, Busan, Korea, 1-4 November 2015.

16. Zhao, C.; Wood, G.S.; Xie, J.; Chang, H.; Pu, S.; Kraft, M. A force sensor based on three weakly coupled resonators with ultrahigh sensitivity. Sens. Actuators A Phys. 2015, 232, 151-162.

17. Sawada, R.; Higurashi, E.; Ito, T.; Ishikawa, I. An accelerometer incorporating a laser microencoder for a wide measurable range. Sens. Actuators A Phys. 2007, 136, 161-167.

18. Łuczak, S.; Grepl, R.; Bodnicki, M. Selection of mems accelerometers for tilt measurements. J. Sens. 2017, $2017,9796146$.

19. Łuczak, S. Erratum to: Guidelines for tilt measurements realized by MEMS accelerometers. Int. J. Precis. Eng. Manuf. 2014, 15, 2011-2019.

20. Li, E.; Zhong, J.; Chang, H. Tilt sensor based on a dual-axis microaccelerometer with maximum sensitivity and minimum uncertainty in the full measurement range. IET Micro Nano Lett. 2017, 12, 866-870.

21. Wang, S.; Ren, J.; Zhang, T.; Weng, Y.; Jiang, Z.; Wei, X. A MEMS resonant tilt sensor with high sensitivity maintained in the whole $360^{\circ}$ measurement range. In Proceedings of the 2016 IEEE on SENSORS, Orlando, FL, USA, 30 October-3 November 2016.

22. Shin, D.D.; Ahn, C.H.; Chen, Y.; Christensen, D.L.; Flader, I.B.; Kenny, T.W. Environmentally robust differential resonant accelerometer in a wafer-scale encapsulation process. In Proceedings of the 2017 IEEE 30th International Conference on MICRO Electro Mechanical Systems, Las Vegas, NV, USA, 22-26 January 2017. 
23. Wei, X.; Seshia, A.A. Differential piezoresistive sensing in a bulk-mode micromechanical resonator. IET Micro Nano Lett. 2013, 8, 107-110.

24. Antonio, D.; Zanette, D. H.; López, D. Frequency stabilization in nonlinear micromechanical oscillators. Nat. Commun. 2012, 3, 806.

25. El-Sheimy, N.; Hou, H.; Niu, X. Analysis and modeling of inertial sensors using Allan variance. IEEE Trans. Instrum. Meas. 2008, 57, 140-149.

26. Chui, C.; Chen, G. Kalman Filtering With Real-Time Applications, 4th ed.; Springer: Berlin, Germany, 2009.

27. Li, B.; Zhang, H.; Zhong, J.; Chang, H. A mode localization based resonant MEMS tilt sensor with a linear measurement range of $360^{\circ}$. In Proceedings of the 2016 IEEE 29th International Conference on MICRO Electro Mechanical Systems, Shanghai, China, 24-28 January 2016; pp. 938-941.

(C) 2018 by the authors. Licensee MDPI, Basel, Switzerland. This article is an open access article distributed under the terms and conditions of the Creative Commons Attribution (CC BY) license (http:// creativecommons.org/licenses/by/4.0/). 